involved in procedures performed on both groups of patients, this explanation cannot account for the increased SSI rates observed primarily in group I patients. Lastly, we also cannot entirely exclude the possibility of our findings occurring solely as an artifact of our particular data set. Confirmation in other independent data sets is needed before broad generalizations can be made.

In conclusion, while elimination of preoperative hospital stays has been embraced widely as safe and cost-effective for many surgical procedures, SSI rates of procedures performed in this setting have not been critically evaluated. The results of our study suggests that elimination of preoperative hospital stay is not necessarily without adverse consequence to the patient. Specifically, for certain procedures, patients may be placed at higher-than-expected risk of SSI by undergoing elective surgery on the day of their admission. Further study of factors associated with higher-than-expected SSI rates in these patients is needed.

\section{REFERENCES}

1. Selwyn S. Skin preparation, the surgical 'scrub' and related rituals. In: Karran S, ed. Controversies in Surgical Sepsis. Eastbourne and New York: Praeger; 1980:23-32.

2. Horan TC, Gaynes RP, Martone WJ, Jarvis WR, Emori TG. CDC definitions of nosocomial surgical site infections, 1992: a modification of CDC definitions of surgical wound infections. Infect Control Hosp Epidemiol 1992;13:606-608.

3. Garner JS. Guidelines for prevention of surgical wound infections, 1985. Infect Control Hosp Epidemiol 1986;7:193-200.

4. Manian FA, Meyer L. Adjunctive use of monthly physician questionnaires for surveillance of surgical site infections after hospital discharge and in ambulatory surgery patients: report of a seven-year experience. Am J Infect Control 1997;25:390-394.

5. Owens WD, Felts JA, Spitznagel EL. ASA physical status classification: a study of consistency of ratings. Anesthesiology 1978;49:239-243

6. Culver DH, Horan TC, Gaynes RP, Martone WJ, Jarvis WR, Emori TG, et al. Surgical wound infection rates by wound class, operative procedure, and patient risk index. Am J Med 1991;91(suppl 3D):152-157.

\title{
Pseudomonas Infections in Children With Cystic Fibrosis
}

\section{Gina Pugliese, RN, MS Martin S. Favero, $\mathbf{P h D}$}

Investigators from the University of Wisconsin Medical School in Madison have reported a study of Pseudomonas aeruginosa infections in children with cystic fibrosis (CF). The objective was to determine whether patients diagnosed through neonatal screening and treated in early infancy were more likely to become colonized with $P$ aeruginosa compared with those identified by standard diagnostic methods. Patients were managed with a standardized evaluation and treatment protocol at two Wisconsincertified CF centers (one urban, one not). Overall, there were no differences in acquisition of respiratory pathogens between the screened and the control (standard diagnosis) groups. However, experiences at the two centers differed significantly.

The median Pseudomonas-free survival period in the screened group was 52 weeks at the urban center, contrasted with 289 weeks in the other center. In addition, assessment of data for the entire $\mathrm{CF}$ populations showed a significantly higher prevalence of $P$ aeruginosa colonization at the urban center in patients between the ages of 3 and 9 years.

The urban center differed from the other center not only by location but also by following patients with the standard US approach, in which newly diagnosed young children were interspersed with older $\mathrm{CF}$ patients, and by having more opportunities for social interactions among the $\mathrm{CF}$ patients.

These results present questions and generate hypotheses on risk factors for acquisition of $P$ aeruginosa in $\mathrm{CF}$ and suggest that clinic exposures or social interactions may predispose such patients to Pseudomonas infections.

FROM: Farrell PM, Shen G, Splaingard M, et al. Acquisition of Pseudomonas aeruginosa in children with cystic fibrosis. Pediatrics 1997;100:E2. 